\title{
Chiral Triptycenes Owing their Chirality to Hindered Rotation of a Bridgehead Substituent. Crystal Structure, Chromatographic Enantiomer Resolution, and Circular Dichroism Spectra
}

\author{
Roland Isaksson, ${ }^{a}$ Michinori Oki, ${ }^{b, *}$ Jan Sandström, ${ }^{c, *}$ M. Rachel Suissa ${ }^{d}$ and Shinji Toyota ${ }^{b}$ \\ a Department of Analytical Pharmaceutical Chemistry, Biomedical Center, Uppsala University, P.0. Box 574, S-751 23 \\ Uppsala, Sweden, ${ }^{b}$ Department of Chemistry, Okayama University of Science, 1-1 Ridaicho, Okayama 700, Japan, \\ ${ }^{c}$ Division of Organic Chemistry 3, Chemical Center, University of Lund, P.O. Box 124, S-221 00 Lund, Sweden and \\ ${ }^{d}$ Department of Organic Chemistry, The Hebrew University of Jerusalem, Jerusalem 91904, Israel
}

\begin{abstract}
Isaksson, R., Oki, M., Sandström, J., Suissa, M. R. and Toyota, S., 1993. Chiral Triptycenes Owing their Chirality to Hindered Rotation of a Bridgehead Substituent. Crystal Structure, Chromatographic Enantiomer Resolution and Circular Dichroism Spectra. - Acta Chem. Scand. 47: 570-576.

The complete enantiomer separation of the $s c$ forms of two methyl 3-(1,4-disubstituted 9-triptycenyl)-3-methylbutanoates by chromatography on swollen microcrystalline triacetylcellulose is described. The structure of the 1,4-dimethyl derivative 1 was determined by X-ray crystallography. The triptycene part was found to be nearly symmetrical with almost planar benzene rings, and the ester group was rotated away from the 1-methyl group. The structure of the global energy minimum calculated by the empirical force-field MM2-87 was similar to the structure in the crystal. The CD spectra of the first eluted enantiomers were recorded and compared with spectra calculated by a semiempirical method, using monopolar transition charges from $\mathrm{CNDO} / \mathrm{S}$ calculations. The calculations show that rotational strengths of the right order of magnitude may arise through interactions between the $n \rightarrow \pi^{*}$ and $\pi \rightarrow \pi^{*}$ transitions in the ester group and the $\pi \rightarrow \pi^{*}$ transitions in the benzene rings. However, the calculated spectra show only partial resemblance with the experimental ones, and no safe conclusions regarding absolute configurations are possible.
\end{abstract}

Optically active triptycenes and their analogues have been the subject of much interest in the past, ${ }^{1-5}$ e.g., to clarify stereochemical relations and to demonstrate the effects of through-bond and through-space electronic interactions in the generation of rotational strength in the electronic transitions. In general, the chirality has been the result of unequal substitution or annellation in the triptycene rings, rendering the triptycene unit dissymmetric. Only two cases are known in which the triptycene or triptycene analogue moiety has a local plane of symmetry, and the chirality is the result of hindered rotation of the bridgehead substituent. In 1975, Yamamoto et al. ${ }^{6}$ described the resolution of the racemic synclinal $(s c)$ form of 9-(1,1-dimethyl-2-phenylethyl)-11-methoxycarbonyl9,10-dihydro-9,10-ethenoanthracene-12-carboxylic acid as its (-)-menthyl ester. The enthalpy barrier to exchange between the $s c$ and achiral antiperiplanar (ap) forms was determined by thermal equilibration in the temperature range $111-152^{\circ} \mathrm{C}$ to be $135.6 \mathrm{~kJ} \mathrm{~mol}^{-1}$. In 1986, Schwartz et al. ${ }^{7}$ described the synthesis of optically

\footnotetext{
* To whom correspondence should be addressed.
}

active $9,9^{\prime}$-bitriptycenyl-2,2'-dicarboxylic acid ( $s c$ form), which showed no isomerization to the ap form at $300^{\circ} \mathrm{C}$, indicating a barrier to rotation about the $9,9^{\prime}$ bond higher than $230 \mathrm{~kJ} \mathrm{~mol}^{-1}$.

One of us has described the synthesis of 3-(1,4-disubstituted-9-triptycenyl)-3-methylbutanoic acids and derivatives thereof. ${ }^{8}$ These compounds have been separated into achiral ap and ( \pm )-sc forms. We now report the enantiomer resolution of the $s c$ forms of the methyl esters (1 and 2) of the 1,4-dimethyl and 1,4-dimethoxy acids, their CD spectra, and the crystal structure of 1 .

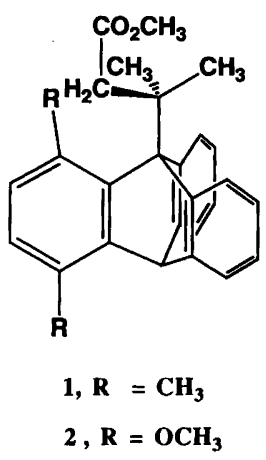


Chromatographic techniques for enantiomer resolution have developed greatly in recent years, ${ }^{9,10}$ and we have used the versatile technique with swollen microcrystalline triacetylcellulose $(\mathrm{TAC})^{11,12}$ as the chiral stationary phase (CSP).

\section{Experimental}

The preparation of the carboxylic esters 1 and 2 has been described. ${ }^{8}$ The enantiomer resolutions, with $95 \%$ aqueous ethanol as the mobile phase, were performed with the equipment already described. ${ }^{13}$ 1,3,5-Tri-tertbutylbenzene was used as the non-retained reference. ${ }^{14}$ Samples of ca. $0.5 \mathrm{mg}$ of 1 and 2 were dissolved in the minimum amount of ethanol $(2-5 \mathrm{ml})$ and injected into the chromatographic system with a flow rate of $1 \mathrm{ml} \mathrm{min}^{-1}$. The capacity and selectivity factors ${ }^{15}$ for $\mathbf{1}$ and $\mathbf{2}$ are found in Table 1 . Owing to low efficiency ${ }^{12}$ and relatively small selectivity factors a recycling technique ${ }^{13}$ was used in the preparative enantiomer separation. The 1,4-dimethyl compound 1 gave reasonable separation, and after three chromatographic cycles baseline separation was achieved. The 1,4-dimethoxy analogue 2 was less well resolved, but pure $(+)$ and $(-)$-forms could be obtained by recycling twice early and late fractions in the first chromatogram while discarding the intermediate fractions and repeating this process over again. The purity of the enantiomers of 2 was established by recording the ${ }^{1} \mathrm{H}$ NMR spectra $(300 \mathrm{MHz})$ of samples of racemic and resolved material in $\mathrm{CDCl}_{3}$ containing one molar equivalent of the chiral shift reagent $\mathrm{Eu}(\mathrm{hfbc})_{3} .{ }^{16}$ The racemic material gave a spectrum with two sets of equally intense $\mathrm{H}-2, \mathrm{H}-3$ resonances, whereas the resolved materials gave only one set $($ e.e. $\geqslant 0.98)$.

$C D$ Spectra. The CD spectra were recorded with a JASCO Model J-500A spectropolarimeter directly on the collected chromatographic fractions. The concentrations were monitored by means of UV spectra, which were recorded on a Cary Model 2290 spectrophotometer using $0.1 \mathrm{~cm}$ cells, which permits measurements down to $192 \mathrm{~nm}$ without significant stray light.

Table 1. Capacity and selectivity factors for 1 and 2 in $95 \%$ aqueous ethanol.

\begin{tabular}{lllll}
\hline Compound & $k_{1}^{\prime a}$ & $k_{2}^{\prime a}$ & $a^{a}$ & {$[a]_{364}^{b}$} \\
\hline $\mathbf{1}$ & 0.96 & 1.61 & 1.68 & + \\
$\mathbf{2}$ & 0.88 & 1.00 & 1.14 & +
\end{tabular}

a The capacity factors for the first and second eluted enantiomer, $k_{1}^{\prime}$ and $k_{2}^{\prime}$, and the selectivity factors a were calculated by eqns. (T1) and (T2), ${ }^{15}$ where $t_{\mathrm{i}}$ and $t_{0}$ are the retention times for enantiomers and non-retained reference respectively.

$k_{\mathrm{i}}^{\prime}=\left(t_{\mathrm{i}}-t_{\mathrm{o}}\right) / t_{\mathrm{o}} \quad(i=1$ and 2$)$

$\mathrm{a}=k_{2}^{\prime} / k_{1}^{\prime}$

${ }^{b}$ Sign of rotation of first eluted enantiomer.
$X$-Ray crystallography. The experimental details in the crystallographic study of $( \pm)-s c-1$ are presented in Table 2, fractional positional parameters in Table 3, and selected bond lengths, bond angles, and torsional angles in Table 4 . The structure obtained by the calculation was (-)-sc-1 ( $M$ helicity). The crystals were grown from hexane-dichloromethane.

CNDO/S calculations. These were performed with the program described by Guimon et al ${ }^{17}$ with configuration interaction between the 99 lowest singly excited configurations. The two-centre Coulomb integrals were calculated by the method of Nishimoto and Mataga. ${ }^{18}$ The empirical force-field calculations were performed with the Allinger MM2-87 force field, ${ }^{19,} 20$ in conjunction with the Macintosh II molecular-modelling program MacMimic. ${ }^{21}$

Calculations of $C D$ and $U V$ spectra. These were performed by using the matrix program developed by Schellman and coworkers. ${ }^{22,23}$ This program is based on interactions between transition fields in molecules containing two or more chirally disposed achiral chromophores. The rotational strengths of the transitions involved are generated by the coupled oscillator, ${ }^{24}$ the

Table 2. Physical properties and parameters for the data collection and refinement of $\pm(s c)-1$.

\begin{tabular}{|c|c|}
\hline $\begin{array}{l}\text { Volume of unit cell } \\
\text { Crystal system } \\
\text { Space group } \\
Z \text { value } \\
\text { Densities: } d_{\text {obs }} ; d_{\text {calc }} \\
F(000) \\
\text { Linear absorption coefficient } \\
\text { Diffractometer used } \\
\text { Radiation } \\
\text { Maximum sin }(\theta) / \lambda \\
\text { Total reflections measured } \\
\text { Unique reflections } \\
\text { Internal consistency: } R_{\text {int }} \\
\text { Function minimized was } \\
\left.\left.\text { sum [w }\left|F_{0}\right|^{2}-\left|F_{\mathrm{c}}\right|^{2}\right)^{2}\right] \text { with } \\
\text { W }=1.0 /\left[\left(\sigma\left|F_{0}\right|^{2}+0.0000\left|F_{0}\right|^{2}\right]\right. \\
\text { Reflections used }[F>3.00 \sigma(F)] \\
\text { No. of variables } \\
\text { Residuals: } R ; R_{w} \\
\text { Goodness of fit: S } \\
\text { Maximum shift/e.s.d. in final cycle } \\
\text { Maximum negative peak } \\
\text { in final diff. map } \\
\text { Maximum positive peak } \\
\text { in final diff. map }\end{array}$ & $\begin{array}{l}\mathrm{C}_{28} \mathrm{H}_{28} \mathrm{O}_{2} \\
396.50 \\
0.40 \times 0.30 \times 0.15 \mathrm{~mm}^{3} \\
a=11.673(2) \AA \\
b=17.598(3) \AA \\
c=11.376(2) \AA \\
\mathrm{a}=90.0^{\circ} \\
\beta=113.93(1)^{\circ} \\
\mathrm{Y}=90.0^{\circ} \\
2136.0(7) \AA^{3} \\
\text { monoclinic } \\
P 2{ }_{1} / \mathrm{a}(\mathrm{No} .14) \\
4 \\
1.20 ; 1.23 \mathrm{~g} \mathrm{~cm}-3 \\
848 \\
0.40 / \mathrm{cm}(\mathrm{Mo} \mathrm{Ka}) \\
\mathrm{Mac} \mathrm{Science} \mathrm{MXC18} \\
\mathrm{Mo} \mathrm{Ka}(\lambda=0.71073) \\
0.650 \\
5811 \\
5244 \\
0.04 \\
\end{array}$ \\
\hline
\end{tabular}


Table 3. Positional parameters and equivalent isotropic thermal parameters for 1. E.s.d. in parentheses.

\begin{tabular}{|c|c|c|c|c|}
\hline Atom & $x$ & $y$ & $z$ & $B(\mathrm{eq})$ \\
\hline 01 & $0.6100(2)$ & $-0.0852(2)$ & $0.2569(2)$ & $8.6(1)$ \\
\hline 02 & $0.6751(2)$ & $-0.0147(1)$ & $0.4351(2)$ & $6.34(8)$ \\
\hline C1 & $0.1377(2)$ & $-0.0292(2)$ & $0.1875(2)$ & $3.64(9)$ \\
\hline C2 & $0.0241(3)$ & $-0.0462(2)$ & $0.1947(3)$ & $4.09(9)$ \\
\hline C3 & $-0.0576(3)$ & $0.0071(2)$ & $0.2028(3)$ & $4.3(1)$ \\
\hline C4 & $-0.0300(2)$ & $0.0841(2)$ & $0.2016(2)$ & $3.58(9)$ \\
\hline $\mathrm{C} 4 \mathrm{a}$ & $0.0830(2)$ & $0.1021(1)$ & $0.1960(2)$ & $3.19(8)$ \\
\hline C5 & $0.2612(3)$ & $0.2462(2)$ & $0.4074(3)$ & $4.4(1)$ \\
\hline C6 & $0.3782(3)$ & $0.2512(2)$ & $0.5082(3)$ & $5.2(1)$ \\
\hline C7 & $0.4733(3)$ & $0.2049(2)$ & $0.5086(3)$ & $4.6(1)$ \\
\hline C8 & $0.4530(3)$ & $0.1523(2)$ & $0.4115(3)$ & $4.0(1)$ \\
\hline $\mathrm{C8a}$ & $0.3362(2)$ & $0.1453(1)$ & $0.3093(2)$ & $3.15(8)$ \\
\hline c9 & $0.2937(2)$ & $0.0906(1)$ & $0.1894(2)$ & $3.01(7)$ \\
\hline $\mathrm{C9a}$ & $0.1721(2)$ & $0.0482(1)$ & $0.1914(2)$ & $3.06(8)$ \\
\hline $\mathrm{C} 10$ & $0.1199(2)$ & $0.1844(1)$ & $0.1950(2)$ & $3.44(9)$ \\
\hline $\mathrm{C} 10 \mathrm{a}$ & $0.2425(2)$ & $0.1942(1)$ & $0.3092(2)$ & $3.40(8)$ \\
\hline C11 & $0.1455(2)$ & $0.1946(1)$ & $0.0762(2)$ & $3.17(8)$ \\
\hline $\mathrm{C} 12$ & $0.2374(2)$ & $0.1457(1)$ & $0.0715(2)$ & $3.02(8)$ \\
\hline $\mathrm{C} 13$ & $0.2674(2)$ & $0.1520(2)$ & $-0.0357(2)$ & $3.64(9)$ \\
\hline $\mathrm{C} 14$ & $0.2053(3)$ & $0.2045(2)$ & $-0.1333(3)$ & $4.2(1)$ \\
\hline C15 & $0.1125(3)$ & $0.2513(2)$ & $-0.1261(3)$ & $4.2(1)$ \\
\hline C16 & $0.0829(3)$ & $0.2462(2)$ & $-0.0204(3)$ & $3.72(9)$ \\
\hline $\mathrm{C} 17$ & $0.2021(3)$ & $-0.1019(2)$ & $0.1736(4)$ & $6.1(1)$ \\
\hline $\mathrm{C} 18$ & $-0.1218(3)$ & $0.1431(2)$ & $0.2057(4)$ & $5.6(1)$ \\
\hline C19 & $0.4056(2)$ & $0.0416(1)$ & $0.1854(2)$ & $3.28(8)$ \\
\hline $\mathrm{C} 20$ & $0.5121(3)$ & $0.0951(2)$ & $0.1862(3)$ & $4.0(1)$ \\
\hline $\mathrm{C} 21$ & $0.3632(3)$ & $-0.0075(2)$ & $0.0611(3)$ & $4.1(1)$ \\
\hline $\mathrm{C} 22$ & $0.4598(2)$ & $-0.0117(2)$ & $0.3046(3)$ & $3.91(9)$ \\
\hline $\mathrm{C} 23$ & $0.5870(3)$ & $-0.0426(2)$ & $0.3261(3)$ & $5.0(1)$ \\
\hline $\mathrm{C} 24$ & $0.8018(4)$ & $-0.0428(4)$ & $0.4650(4)$ & $10.0(2)$ \\
\hline $\mathrm{H} 2$ & $0.006(3)$ & $-0.097(2)$ & $0.194(3)$ & $3.81(0)$ \\
\hline H3 & $-0.133(3)$ & $-0.010(2)$ & $0.210(3)$ & $4.04(0)$ \\
\hline H5 & $0.193(3)$ & $0.276(2)$ & $0.407(3)$ & $4.06(0)$ \\
\hline $\mathrm{H} 6$ & $0.389(3)$ & $0.292(2)$ & $0.571(3)$ & $4.91(0)$ \\
\hline $\mathrm{H} 7$ & $0.558(3)$ & $0.212(2)$ & $0.575(3)$ & $4.41(0)$ \\
\hline H8 & $0.519(3)$ & $0.122(2)$ & $0.418(3)$ & $2.94(0)$ \\
\hline $\mathrm{H} 10$ & $0.052(2)$ & $0.216(2)$ & $0.195(3)$ & $3.13(0)$ \\
\hline $\mathrm{H} 13$ & $0.336(2)$ & $0.120(2)$ & $-0.045(3)$ & $3.39(0)$ \\
\hline $\mathrm{H} 14$ & $0.225(2)$ & $0.208(2)$ & $-0.215(3)$ & $3.90(0)$ \\
\hline $\mathrm{H} 15$ & $0.065(3)$ & $0.290(2)$ & $-0.192(3)$ & $4.05(0)$ \\
\hline $\mathrm{H} 16$ & $0.017(3)$ & $0.276(2)$ & $-0.014(3)$ & $3.55(0)$ \\
\hline $\mathrm{H} 17 \mathrm{~A}$ & $0.269(3)$ & $-0.098(2)$ & $0.145(3)$ & $5.55(0)$ \\
\hline$H 17 B$ & $0.240(3)$ & $-0.119(2)$ & $0.275(3)$ & $5.55(0)$ \\
\hline $\mathrm{H} 17 \mathrm{C}$ & $0.144(3)$ & $-0.139(2)$ & $0.144(3)$ & $5.55(0)$ \\
\hline $\mathrm{H} 18 \mathrm{~A}$ & $-0.184(3)$ & $0.118(2)$ & $0.222(3)$ & $5.12(0)$ \\
\hline $\mathrm{H} 18 \mathrm{~B}$ & $-0.088(3)$ & $0.177(2)$ & $0.274(3)$ & $5.12(0)$ \\
\hline $\mathrm{H} 18 \mathrm{C}$ & $-0.143(3)$ & $0.174(2)$ & $0.134(3)$ & $5.12(0)$ \\
\hline $\mathrm{H} 20 \mathrm{~A}$ & $0.576(3)$ & $0.104(2)$ & $0.266(3)$ & $3.72(0)$ \\
\hline $\mathrm{H} 20 \mathrm{~B}$ & $0.483(3)$ & $0.149(2)$ & $0.155(3)$ & $3.72(0)$ \\
\hline $\mathrm{H} 20 \mathrm{C}$ & $0.554(2)$ & $0.072(2)$ & $0.130(3)$ & $3.72(0)$ \\
\hline H21A & $0.407(3)$ & $-0.060(2)$ & $0.084(3)$ & $3.78(0)$ \\
\hline H21B & $0.266(3)$ & $-0.017(2)$ & $0.021(3)$ & $3.78(0)$ \\
\hline $\mathrm{H} 21 \mathrm{C}$ & $0.393(3)$ & $0.013(2)$ & $0.001(3)$ & $3.78(0)$ \\
\hline $\mathrm{H} 22 \mathrm{~A}$ & $0.461(3)$ & $0.013(2)$ & $0.384(3)$ & $3.65(0)$ \\
\hline $\mathrm{H} 22 \mathrm{~B}$ & $0.401(2)$ & $-0.061(2)$ & $0.292(3)$ & $3.65(0)$ \\
\hline $\mathrm{H} 24 \mathrm{~A}$ & $0.832(4)$ & $0.032(3)$ & $0.471(4)$ & $9.82(0)$ \\
\hline $\mathrm{H} 24 \mathrm{~B}$ & $0.826(4)$ & $-0.084(3)$ & $0.416(4)$ & $9.82(0)$ \\
\hline $\mathrm{H} 24 \mathrm{C}$ & $0.835(5)$ & $-0.041(3)$ & $0.528(4)$ & $9.82(0)$ \\
\hline
\end{tabular}

Table 4. Intramolecular distances, valence angles and torsion angles in 1. E.s.d. in parentheses.

\begin{tabular}{|c|c|c|c|}
\hline & Distance $/ \AA$ & & Distance $/ \AA$ \\
\hline $\begin{array}{l}\mathrm{O} 1-\mathrm{C} 23 \\
\mathrm{O} 2-\mathrm{C} 23 \\
\mathrm{O} 2-\mathrm{C} 24 \\
\mathrm{C} 1-\mathrm{C} 2 \\
\mathrm{C} 1-\mathrm{C} 9 \mathrm{a} \\
\mathrm{C} 1-\mathrm{C} 17 \\
\mathrm{C} 2-\mathrm{C} 3 \\
\mathrm{C} 3-\mathrm{C} 4 \\
\mathrm{C} 4-\mathrm{C} 4 \mathrm{a} \\
\mathrm{C} 4-\mathrm{C} 18 \\
\mathrm{C} 4 \mathrm{a}-\mathrm{C} 9 \mathrm{a} \\
\mathrm{C} 4 \mathrm{a}-\mathrm{C} 10 \\
\mathrm{C} 5-\mathrm{C} 6 \\
\mathrm{C} 5-\mathrm{C} 10 \mathrm{a} \\
\mathrm{C} 6-\mathrm{C} 7 \\
\mathrm{C} 7-\mathrm{C} 8\end{array}$ & $\begin{array}{l}1.193(5) \\
1.342(3) \\
1.462(5) \\
1.393(4) \\
1.415(4) \\
1.525(4) \\
1.367(5) \\
1.394(4) \\
1.384(4) \\
1.506(5) \\
1.425(4) \\
1.511(4) \\
1.385(4) \\
1.392(4) \\
1.375(5) \\
1.387(4)\end{array}$ & $\begin{array}{l}\text { C8-C8a } \\
\text { C8a-C10a } \\
\text { C8a-C9 } \\
\text { C9-C12 } \\
\text { C9-C19 } \\
\text { C9-C9a } \\
\text { C10-C10a } \\
\text { C10-C11 } \\
\text { C11-C16 } \\
\text { C11-C12 } \\
\text { C12-C13 } \\
\text { C13-C14 } \\
\text { C14-C15 } \\
\text { C15-C16 } \\
\text { C19-C21 } \\
\text { C19-C20 } \\
\text { C19-C22 } \\
\text { C22-C23 }\end{array}$ & $\begin{array}{l}1.392(3) \\
1.391(4) \\
1.576(3) \\
1.568(3) \\
1.582(4) \\
1.611(4) \\
1.502(3) \\
1.509(4) \\
1.384(3) \\
1.392(4) \\
1.404(4) \\
1.398(4) \\
1.389(5) \\
1.382(5) \\
1.556(4) \\
1.557(4) \\
1.557(4) \\
1.506(5)\end{array}$ \\
\hline & Angle $/^{\circ}$ & & Angle $/^{\circ}$ \\
\hline $\begin{array}{l}\mathrm{C} 23-\mathrm{O} 2-\mathrm{C} 24 \\
\mathrm{C} 2-\mathrm{C} 1-\mathrm{C} 9 \mathrm{a} \\
\mathrm{C} 2-\mathrm{C} 1-\mathrm{C} 17 \\
\mathrm{C} 9 \mathrm{a}-\mathrm{C} 1-\mathrm{C} 17 \\
\mathrm{C} 2-\mathrm{C} 3-\mathrm{C} 4 \\
\mathrm{C} 4 \mathrm{a}-\mathrm{C} 4-\mathrm{C} 3 \\
\mathrm{C} 4 \mathrm{a}-\mathrm{C} 4-\mathrm{C} 18 \\
\mathrm{C} 3-\mathrm{C} 4-\mathrm{C} 18 \\
\mathrm{C} 4-\mathrm{C} 4 \mathrm{a}-\mathrm{C} 9 \mathrm{a} \\
\mathrm{C} 4-\mathrm{C} 4 \mathrm{a}-\mathrm{C} 10 \\
\text { C9a-C4a-C10 } \\
\text { C7-C6-C5 } \\
\text { C6-C7-C8 } \\
\text { C7-C8-C8a } \\
\text { C10a-C8a-C8 } \\
\text { C10a-C8a-C9 } \\
\text { C8-C8a-C9 } \\
\text { C12-C9-C8a } \\
\text { C12-C9-C19 } \\
\text { C12-C9-C9a } \\
\text { C8a-C9-C19 } \\
\text { C21-C19-C22 } \\
\text { C21-C19-C9 } \\
\text { C20-C19-C22 }\end{array}$ & $\begin{array}{l}114.5(3) \\
118.0(3) \\
110.3(3) \\
131.7(3) \\
119.7(3) \\
116.9(3) \\
123.1(3) \\
120.0(3) \\
125.0(2) \\
120.0(2) \\
115.0(2) \\
119.2(3) \\
121.1(2) \\
121.2(3) \\
116.7(2) \\
113.9(2) \\
129.4(2) \\
103.8(2) \\
112.1(2) \\
103.4(2) \\
112.6(2) \\
108.9(2) \\
111.5(2) \\
108.6(2)\end{array}$ & $\begin{array}{l}\text { C20-C19-C9 } \\
\text { C22-C19-C9 } \\
\text { C8a-C9-C9a } \\
\text { C19-C9-C9a } \\
\text { C1-C9a-C4a } \\
\text { C1-C9a-C9 } \\
\text { C4a-C9a-C9 } \\
\text { C23-C22-C19 } \\
\text { O1-C23-O2 } \\
\text { O1-C23-C22 } \\
\text { O2-C23-C22 } \\
\text { C10a-C10-C11 } \\
\text { C10a-C10-C4a } \\
\text { C11-C10-C4a } \\
\text { C8a-C10a-C5 } \\
\text { C8a-C10a-C10 } \\
\text { C5-C10a-C10 } \\
\text { C16-C11-C12 } \\
\text { C16-C11-C10 } \\
\text { C12-C11-C10 } \\
\text { C11-C12-C13 } \\
\text { C11-C12-C9 } \\
\text { C13-C12-C9 } \\
\text { C21-C19-C20 }\end{array}$ & $\begin{array}{l}109.7(2) \\
110.6(2) \\
104.0(2) \\
119.4(2) \\
116.1(2) \\
133.3(2) \\
110.6(2) \\
112.6(3) \\
123.1(3) \\
126.2(3) \\
110.7(3) \\
107.2(2) \\
106.1(2) \\
106.4(2) \\
122.6(2) \\
113.8(2) \\
123.6(2) \\
122.8(3) \\
123.8(3) \\
113.3(2) \\
116.9(2) \\
114.3(2) \\
128.8(2) \\
107.4(3)\end{array}$ \\
\hline
\end{tabular}

Torsion angles ${ }^{\circ}$

\begin{tabular}{lrlr}
\hline Benzene ring 1 & \multicolumn{3}{l}{ Benzene ring 2 } \\
C1-C2-C3-C4 & 1.3 & C5-C6-C7-C8 & -1.5 \\
C2-C3-C4-C4a & -2.0 & C6-C7-C8-C8a & 0.9 \\
C3-C4-C4a-C9a & 0.0 & C7-C8-C8a-C10a & 0.8 \\
C4-C4a-C9a-C1 & 2.4 & C8-C8a-C10a-C5 & -2.0 \\
C4a-C9a-C1-C2 & -3.0 & C8a-C10a-C5-C6 & 1.5 \\
C9a-C1-C2-C3 & 1.3 & C10a-C5-C6-C7 & 0.3 \\
& & & \\
Benzene ring 3 & & -COOMe & \\
C11-C12-C13-C14 & -1.1 & C9a-C9-C19-C22 & $-59.5-\mathrm{sc}$ \\
C12-C13-C14-C15 & -0.1 & C9-C19-C22-C23 & -163.6 anti \\
C13-C14-C15-C16 & 0.8 & C19-C22-C23-O1 & -66.1 \\
C14-C15-C16-C11 & -0.2 & C19-C22-C23-O2 & 112.9 \\
C15-C16-C11-C12 & -1.0 & O1-C23-O2-C24 & $-2.0 \mathrm{~s}-$ cis \\
C16-C11-C12-C13 & 1.6 & C22-C23-O2-C24 & 178.9 \\
\end{tabular}


magnetic electric $(m-\mu)$ coupling, ${ }^{25,26}$ and the oneelectron $^{27}$ mechanisms. The input consists of transition energies, electric and magnetic transition moments, transition charge densities (transition monopoles and quadrupoles), and, for the one-electron mechanism, the ground-state electronic charge density. The program calculates dipole and rotational strengths of all resulting transitions and CD and UV spectra based on the Gaussian shape of the individual bands.

The input for 1 and 2 consists of the data for the ${ }^{1} \mathbf{L}_{\mathrm{b}},{ }^{1} \mathbf{L}_{\mathrm{a}},{ }^{1} \mathbf{B}_{\mathrm{b}}$, and ${ }^{1} \mathbf{B}_{\mathrm{a}}$ transitions ${ }^{28}$ in the aromatic chromophores, and for the $\mathrm{n} \rightarrow \pi^{*}$ and $\pi \rightarrow \pi^{*}$ transitions in the ester group. ${ }^{29}$ Transition energies and transition moments were obtained from experimental UV spectra of $o$-xylene and 1,2,3,4-tetramethylbenzene. As 1,4dimethoxy-2,3-dimethylbenzene was not available, the data were taken from 1,4-dimethoxybenzene and adjusted slightly on the basis of the differences between this molecule and its 2,3-dimethyl derivative obtained by $\mathrm{CNDO} / \mathrm{S}$ calculations. The input for the ester group is based on the vacuum-UV gas-phase spectrum of ethyl acetate described by Nagakura et al. ${ }^{30} \mathrm{~A}$ band, assigned to the first $\pi \rightarrow \pi^{*}$ transition, was observed at $164 \mathrm{~nm}$ $(\varepsilon=3600)$. The change from gas phase to solution is normally accompanied by substantial bathochromic shifts, and the wavelength given above was corrected with the shift observed for $N, N$-dimethylformamide from the gas phase to ethanol solution $\left(2400 \mathrm{~cm}^{-1}\right)^{31}$ to give $\lambda_{\max }=171 \mathrm{~nm}$. With the assumption of an exponential halfwidth $\Delta$ (half bandwidth at $1 / \mathrm{e}$ of maximum intensity, $\varepsilon_{\text {max }}$ ) of $6 \mathrm{~nm}$, a transition moment $(\mu)$ of $1.43 \mathrm{D}$ was calculated by eqn. (1), which is based on an assumed

$\mu=0.1276\left(\frac{\Delta \cdot \varepsilon_{\max }}{\lambda}\right)^{1 / 2}$

Gaussian shape of the absorption band. The $n \rightarrow \pi^{*}$ transition was placed at $207 \mathrm{~nm}$ according to the work of Closson and Haug. ${ }^{32}$ Transition monopoles were obtained by CNDO/S calculations and scaled to conform with the experimental transition moments.

\section{Results and discussion}

The UV spectra of triptycene and its derivatives have been the subject of several studies over the years. An early study ${ }^{33}$ invoked single bond-no bond resonance to explain the bathochromic and hyperchromic shifts caused by interaction between the three benzene rings. However, there now seems to be general acceptance that the triptycene spectrum is qualitatively explained by throughspace (exciton) interaction between the local transition moments in the benzene rings. ${ }^{2,34,35} \mathrm{CD}$ spectra of benzo- and naphtho-annellated triptycenes have been rationalized by the same model, ${ }^{5,36}$ but they have also been reproduced by $\pi$-electron SCF calculations including an inter-ring (homoconjugation) resonance integral $\beta_{\mathrm{hc}}=0.32 \beta_{\mathrm{arom}}{ }^{37}$ In triptycenes containing strong acceptor groups, charge-transfer transitions become important. $^{38}$

The UV spectrum of triptycene displays one band with vibrational fine structure at $270 \mathrm{~nm}(\varepsilon=4650)$ and two strong bands at 214 and $196 \mathrm{~nm}(\varepsilon=63000){ }^{2}$ The UV spectra of 1 and $\mathbf{2}$ are fairly similar to that of triptycene, although the long-wavelength band of $\mathbf{1}$ is weaker, and that of $\mathbf{2}$ is split into two, one at 296 and one at $278 \mathrm{~nm}$, the former being assigned to the dimethoxybenzene chromophore (Fig. 1 and Table 5). A calculation for triptycene by the Schellman matrix method, ${ }^{22,23}$ using the ${ }^{1} \mathrm{~L}_{\mathrm{b}},{ }^{1} \mathrm{~L}_{\mathrm{a}},{ }^{1} \mathrm{~B}_{\mathrm{b}}$, and ${ }^{1} \mathrm{~B}_{\mathrm{a}}$ transitions in three $o$-xylene chromophores as input, gave 12 transitions, six of which were symmetry-forbidden. Of the six allowed ones, a degenerate pair $\left({ }^{1} \mathrm{~L}_{\mathrm{b}}\right.$ type, $x, y$-polarized, Fig. 2$)$ fell at $260 \mathrm{~nm}$ (dipole strength $0.62 \mathrm{D}^{2}$ for each). A strong $z$-polarized transition (combination of ${ }^{1} \mathrm{~L}_{\mathrm{a}}$ and ${ }^{1} \mathrm{~B}_{\mathrm{a}}$ transitions, dipole strength $61.5 \mathrm{D}^{2}$ ) was predicted at $197 \mathrm{~nm}$, and a degenerate pair of $x, y$-polarized transitions $\left({ }^{1} \mathbf{B}_{\mathrm{b}}\right.$ combinations, dipole strength $26.2 \mathrm{D}^{2}$ for each) at

$$
\varepsilon \times 10^{-3}
$$

(a)

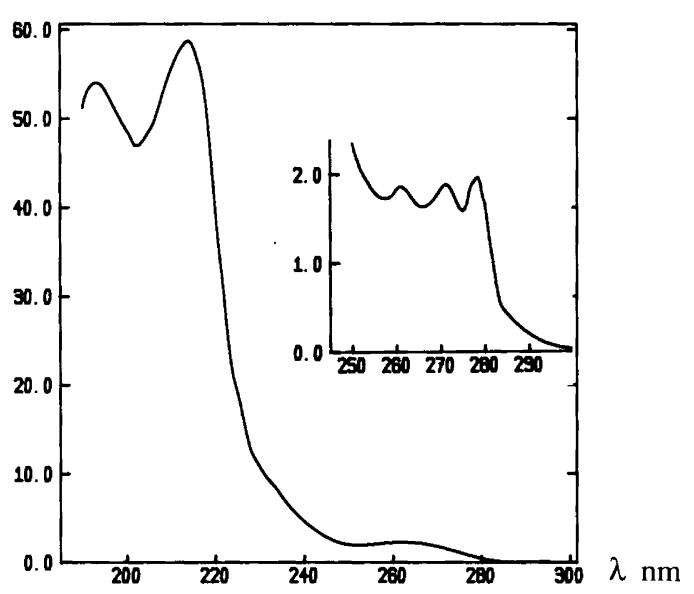

$\varepsilon \times 10^{-3}$

(b)

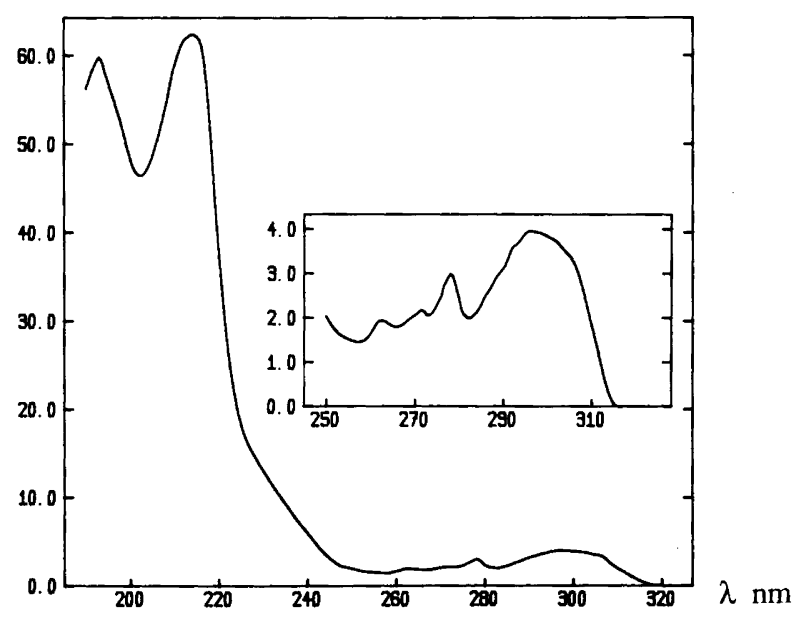

Fig. 1. (a) UV spectrum of 1 in ethanol ( $\epsilon$ in units of $\mathrm{cm}^{-1} \mathrm{~mol}^{-1} \mathrm{I}$ ); (b) UV spectrum of 2 in ethanol. 
Table 5. UV and CD spectra of 1 and 2 in ethanol. $\epsilon$ and $\Delta \epsilon$ are in units of $\mathrm{M}^{-1} \mathrm{~cm}^{-1}$

\begin{tabular}{lll}
\hline Compound & UV: $\lambda_{\text {max }} / \mathrm{nm}(\epsilon)$ & $\mathrm{CD}: \lambda_{\max } / \mathrm{nm}(\Delta \epsilon)^{a}$ \\
\hline 1 & $278(1950), 271$ & $304(-0.10), 294.5$ \\
$(1860), 214(58800)$, & $(-0.84), 288$ \\
$193(54000)$ & $(+0.51), 279.5$ \\
& $(+3.66), 272.5$ \\
& $(+3.65), 260 \mathrm{~s}$ \\
& $(+7.0), 254(+9.7)$, \\
& $247.5(+10.0), 227$ \\
& $(-12.6), 220$ \\
& $(+3.50), 208$ \\
& & $(-6.4), 197(+20)$ \\
& & $292.5(+0.15), 280$ \\
& & $(+0.61), 274.5$ \\
& & $(+0.29), 262.5$ \\
& & $(-0.54), 256.5$ \\
& $(3000), 271.5$ & $(-0.83), 238$ \\
& $(1940), 214(62300), 262.5$ & $(+1.21), 229$ \\
$193(59700)$ & $(+0.27), 225.5$ \\
& $(+0.18), 219$ \\
& $(-3.05), 211$ \\
& $(+0.54), 199(-4.6)$
\end{tabular}

${ }^{a}$ For the first-eluted enantiomer $\left(E_{1}\right)$.

$194 \mathrm{~nm}$. A third, medium-strong transition $\left(15.4 \mathrm{D}^{2}\right)$ of ${ }^{1} \mathrm{~B}_{\mathrm{a}}$ character $(z$-polarized) was predicted at $152 \mathrm{~nm}$.

A similar calculation for 1,4-dimethoxytriptycene gave 10 allowed transitions: ${ }^{1} \mathrm{~L}_{\mathrm{b}}$ types at $298.5\left(3.81 \mathrm{D}^{2}\right), 260.0$ $\left(0.62 \mathrm{D}^{2}\right)$ and $259.8\left(0.19 \mathrm{D}^{2}\right) \mathrm{nm},{ }^{1} \mathrm{~L}_{\mathrm{a}}$ types at 225.8 $\left(2.69 \mathrm{D}^{2}\right)$ and $212.8\left(15.3 \mathrm{D}^{2}\right) \mathrm{nm},{ }^{1} \mathrm{~B}_{\mathrm{b}}$ types at 206.3 $\left(20.4 \mathrm{D}^{2}\right), 194.4\left(26.2 \mathrm{D}^{2}\right)$ and $178.8\left(1.72 \mathrm{D}^{2}\right) \mathrm{nm}$, and ${ }^{1} \mathrm{~B}_{\mathrm{a}}$ types at $200.0\left(29.7 \mathrm{D}^{2}\right)$ and $166.8\left(22.4 \mathrm{D}^{2}\right)$. A calculation on 1,4-dimethyltriptycene gave very similar results, although with the strong ${ }^{1} L_{b}$ transition at

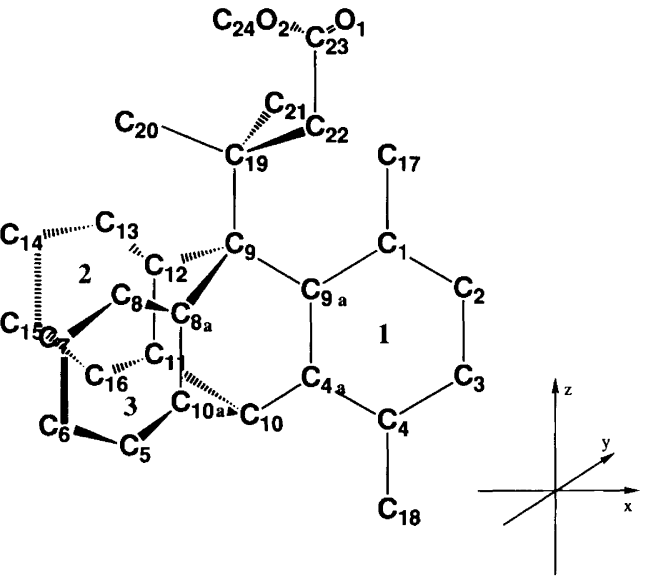

Fig. 2. Numbering of the non-hydrogen atoms in 1 with Cartesian axes.

$289.5 \mathrm{~nm}$ replaced by a weaker one at $277.8 \mathrm{~nm}\left(0.27 \mathrm{D}^{2}\right)$.

The small calculated splittings between the ${ }^{1} \mathrm{~B}$ type bands near $200 \mathrm{~nm}$ show that the coupled oscillator calculation underestimates the interaction between the transitions. A CNDO/S calculation on triptycene predicted weak $x, y$-polarized transitions at 272, 269, and $215 \mathrm{~nm}$, strong $x, y$-polarized transitions at 203.0 and $202.6 \mathrm{~nm}$, and a strong $z$-polarized transition at $184 \mathrm{~nm}$. The last three transitions reproduce the two experimental bands with a moderate wavelength shift. The conclusion seems to be that some electron delocalization is necessary to give a good description of the spectrum of triptycene, as already shown by Harada et al $^{37}$ for substituted triptycenes. However, the coupled oscillator technique was used for semiquantitative calculations of the CD spectra of 1 and 2, since these systems are too large for our $\mathrm{CNDO} / \mathrm{S}$ program.
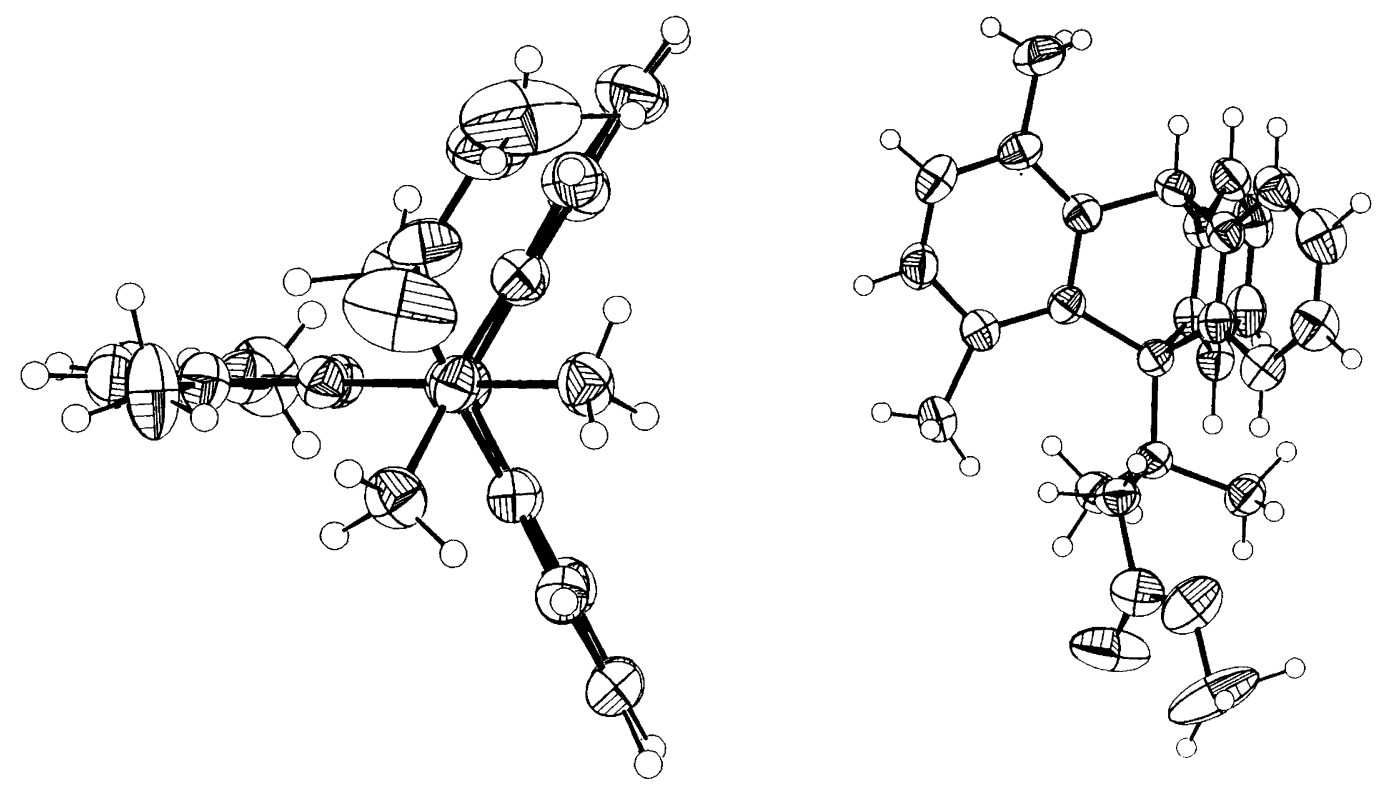

Fig. 3. ORTEP drawings of 1. 
The crystal structure of 1 (Fig. 3) shows nearly planar benzene rings (all torsional angles $<2.5^{\circ}$ ), but the 1-methyl group is strongly bent in the plane of the benzene ring (the $\mathrm{C} 9 \mathrm{a}-\mathrm{Cl}-\mathrm{Cl} 7$ angle is $131.7^{\circ}$ ). The ester group in the bridgehead substituent is, as expected, rotated away from the $\mathrm{C} 1$-methyl group $\left(16^{\circ}\right.$ away from the anti orientation with respect to the C19-C22 bond), with its plane nearly parallel to that of the nearby benzene ring (3).

Force-field calculations on 1 indicated several closelying energy minima. Although some show severely distorted benzene rings, the two with lowest energy have structures with nearly planar benzene rings and are similar to the molecule in the crystal. While this has an $\mathrm{O} 1-\mathrm{C} 23-\mathrm{C} 22-\mathrm{C} 19$ angle of $-66^{\circ}$, the calculated global energy minimum conformation has $110^{\circ}$ (thus the ester group is rotated by nearly $180^{\circ}$ ), and a conformation with $0.8 \mathrm{~kJ} \mathrm{~mol}^{-1}$ higher energy has $-65^{\circ}$. All other angles in the two calculated conformations are quite similar. Thus, the $\mathrm{C} 9 \mathrm{a}-\mathrm{C} 1-\mathrm{C} 17$ angle is $128.9^{\circ}$ and the $\mathrm{C} 19-\mathrm{C} 22$ bond is $35^{\circ}$ off the anti orientation, in the same direction as in the crystal structure. All dihedral angles in the benzene rings are $<2.5^{\circ}$.

Calculations with the structure of 2 gave two similar minimum-energy geometries with nearly planar benzene rings, the same orientation of the ester group with respect to the $\mathrm{C} 19-\mathrm{C} 22$ bond, and a $\mathrm{C} 9 \mathrm{a}-\mathrm{C} 1-\mathrm{O}$ (methoxy) angle of only $121^{\circ}$.

Calculation of the CD spectrum of 1 by the Schellman method using the crystal geometry gave a rather weak spectrum compared with the experimental one. However, this is partly due to the unduly small calculated splitting of the B-type transitions. Analysis of the calculated rotational strengths of the respective transitions reveals that with larger splittings, the calculated strengths of the CD bands would be more similar to the experimental ones, since one transition with a rotational strength corresponding to $\Delta \varepsilon=+4$ is calculated at $197 \mathrm{~nm}$ and one with $\Delta \varepsilon=-10$ at $201 \mathrm{~nm}$. One weaker positive transition is calculated at $210 \mathrm{~nm}$, and several weak positive and negative bands are predicted in the range $233-277 \mathrm{~nm}$. No transition corresponding to the relatively strong negative band at $227 \mathrm{~nm}$ is found in the calculated spectrum.

A calculation based on the geometry of the global minimum-energy conformation of $\mathbf{1}$ gave four fairly strong transitions in the range 196-202 nm, which sum to a negative couplet with $\Delta \varepsilon= \pm 14$. At longer wavelengths, only very weak positive and negative transitions were calculated. A very similar result was obtained for the second lowest conformation, as expected, since the direction of the $\pi \rightarrow \pi^{*}$ transition moment of the ester group is nearly perpendicular to the $\mathrm{C} 23-\mathrm{C} 22$ bond. ${ }^{29} \mathrm{CD}$ spectra of compounds 1 and 2 are shown in Fig. 4.

A similar calculation for 2 based on the MM2-87 energy-minimized geometry gave transitions at 199.5, $206.5,210$, and $212 \mathrm{~nm}$ corresponding to $\Delta \varepsilon=-7,+1.5$, -5 , and +6 , followed by considerably weaker positive and negative transitions.
$\Delta \varepsilon$

(a)

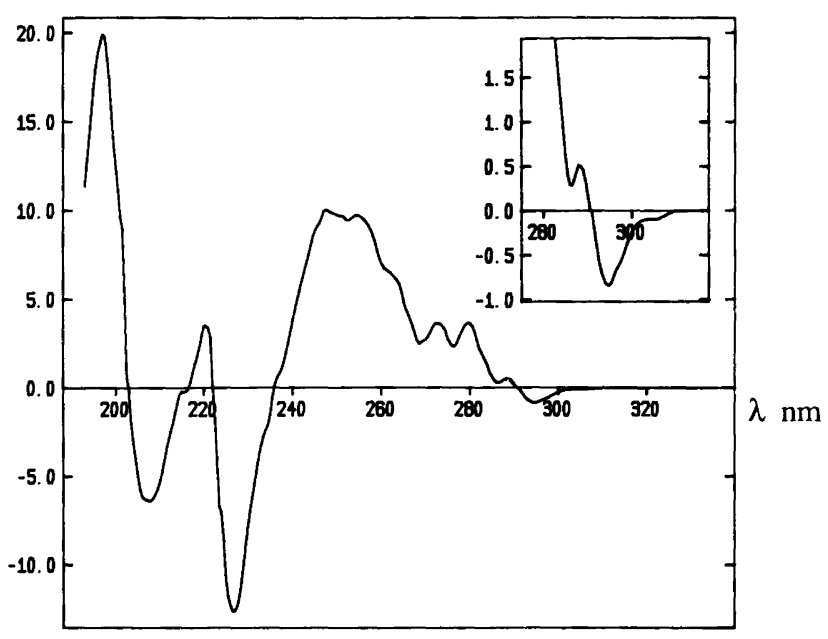

(b)

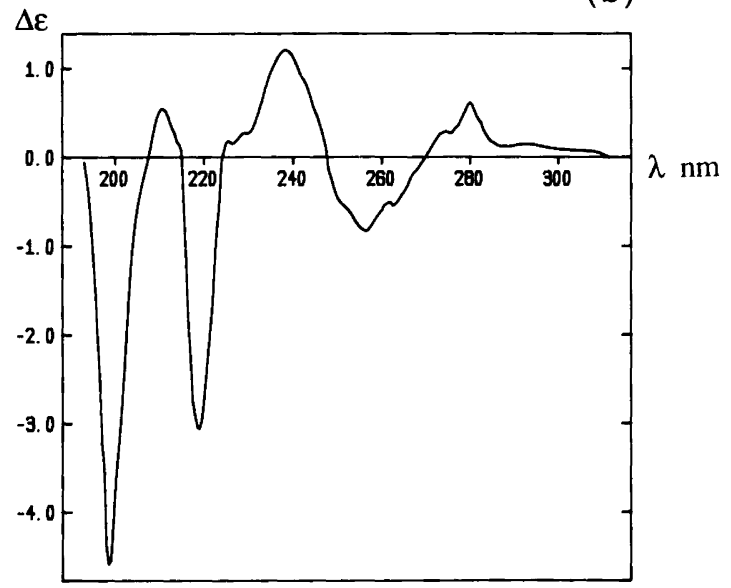

Fig. 4. (a) $C D$ spectrum of $1\left(E_{1}\right)$ in ethanol; (b) $C D$ spectrum of $2\left(E_{1}\right)$ in ethanol.

In summary, it seems that the calculations with geometries based on the $R(M)$ configuration reproduce signs and intensities of the B-type transitions of the first eluted enantiomers of $\mathbf{1}$ and $\mathbf{2}$ reasonably well, but not so with the lower energy transitions. It is therefore not possible to draw any safe conclusions about the absolute configurations. Analysis of the secular equation shows that the rotational strength in the B-region has its main origin in interactions between the ester $\mathrm{n} \rightarrow \pi^{*}$ and $\pi \rightarrow \pi^{*}$ transitions on one side and the B-type transitions in the benzene ring closest to the ester group on the other.

Acknowledgments. Rachel Suissa thanks the Swedish Institute for a maintenance grant. Financial support from the Swedish Natural Science Research Council, the Swedish Board for Technical Development, and the Knut and Alice Wallenberg Foundation to Roland Isaksson and Jan Sandström is gratefully acknowledged. 


\section{References}

1. Kuritani, M., Sakata, Y., Ogura, F. and Nakagawa, M. Bull. Chem. Soc. Jpn. 46 (1973) 605 and earlier work.

2. Tanaka, J., Ozeki-Minakata, K., Ogura, F. and Nakagawa, M. Spectrochim. Acta, Part A 29 (1973) 897.

3. Kaito, A., Tajiri, A., Hatano, M., Ogura, F. and Nakagawa, M. J. Am. Chem. Soc. 98 (1976) 7932.

4. Hezemans, A. M. F. and Groenewege, M. P. Tetrahedron 29 (1973) 1223.

5. Harada, N., Tamai, Y. and Uda, H. J. Org. Chem. 49 (1984) 4266.

6. Yamamoto, G., Nakamura, M. and Oki, M. Bull. Chem. Soc. Jpn. 48 (1975) 2592.

7. Schwartz, L. H., Koukotas, C., Kukkola, P. and Yu, C. S. J. Org. Chem. 51 (1986) 995.

8. Tanaka, T., Yonemoto, K., Nakai, Y., Yamamoto, G. and Oki, M. Bull. Chem. Soc. Jpn. 61 (1988) 3239.

9. Allenmark, S. Chromatographic Enantioseparations: Methods and Applications, 2nd ed., Ellis Horwood, Chichester, UK 1991.

10. Pirkle, W. H. and Pochapsky, T. C. Chem. Rev. 89 (1989) 347.

11. Mannschreck, A., Koller, H. and Wernicke, R. Kontakte (Darmstadt) 1 (1985) 40.

12. Isaksson, R., Erlandsson, P., Hansson, L., Holmberg, A. and Berner, S. J. Chromatogr. 498 (1990) 257.

13. Isaksson, R. and Roschester, J. J. Org. Chem. 50 (1985) 2519.

14. Koller, H., Rimböck, K.-M. and Mannschreck, A J. Chromatogr. 282 (1983) 89.

15. Ref. 9, Section 4.1 .

16. Fraser, R. R., Petit, M. A. and Saunders, J. K. J. Chem. Soc., Chem. Commun. (1971) 1450.

17. Guimon, C., Gonbeau, D. and Pfister-Guillouzo, G. Tetrahedron 29 (1973) 3395.

18. Nishimoto, K. and Mataga, N. Z. Phys. Chem. 12 (1957) 335.

19. Burkert, U. and Allinger, N. L. Molecular Mechanics, ACS
Monograph 177, American Chemical Society, Washington, DC 1982.

20. The program is available from the Quantum Chemistry Program Exchange (University of Indiana, Bloomington, IN 47405) and from Molecular Design Ltd. (San Leandro, CA 94577).

21. The program is available from Intstar Software, IDEON Research Park, S-223 70 Lund, Sweden.

22. Bayley, P. M., Nielsen, E. B. and Schellman, J. A. J. Phys. Chem. 73 (1969) 228.

23. Rizzo, V. and Schellman, J. A. Biopolymers 23 (1984) 435.

24. Kirkwood, J. G. J. Chem. Phys. 5 (1937) 479.

25. Tinoco, I., Jr. Adv. Chem. Phys. 4 (1962) 113.

26. Schellman, J. A. Acc. Chem. Res. 1 (1968) 144.

27. Condon, E. U., Altar, W. and Eyring, H. J. Chem. Phys. 5 (1937) 735.

28. Platt, J. R. J. Chem. Phys. 17 (1949) 484.

29. Roschester, J. and Sandström, J. Tetrahedron 45 (1989) 5081.

30. Nagakura, S., Kaya, K. and Tsubomura, H. J. Mol. Spectrosc. 13 (1964) 1.

31. Basch, H., Robin, M. B. and Kuebler, N. A. J. Chem. Phys. 49 (1969) 5007.

32. Closson, W. D. and Haug, P. J. Am. Chem. Soc. 86 (1969) 2384.

33. Bartlett, P. D. and Lewis, E. S. J. Am. Chem. Soc. 72 (1950) 1005 .

34. Wilcox, C. F., Jr. J. Chem. Phys. 33 (1960) 1874.

35. Wilcox, C. F., Jr. and Craig, A. C. J. Org. Chem. 26 (1961) 2491.

36. Harada, N., Tamai, Y., Takuma, Y. and Uda, H. J. Am. Chem. Soc. 102 (1980) 501

37. Harada, N., Tamai, Y. and Uda, H. J. Am. Chem. Soc. 102 (1980) 506.

38. Harada, N., Uda, H., Nakisuji, K. and Murata, I. J. Chem. Soc., Perkin Trans. 2 (1989) 1449.

Received July 17, 1992. 